\title{
Locating a competitive facility in the plane with a robustness criterion ${ }^{\star}$
}

\author{
R. Blanquero ${ }^{1}$, E. Carrizosa ${ }^{1}$, E.M.T. Hendrix ${ }^{2}$ \\ 1 Facultad de Matemáticas, Universidad de Sevilla, Tarfia s/n, 41012 Sevilla, \\ Spain e-mail: \{rblanquero, ecarrizosa\}@us.es \\ 2 Department of Computer Architecture, University of Málaga, Campus de Teati- \\ nos, 29017, Spain. e-mail: Eligius.Hendrix@wur.nl
}

Received: March 5, 2010/ Accepted: date

\begin{abstract}
A new continuous location model is presented and embedded in the literature on robustness in facility location. The multimodality of the model is investigated, and a branch and bound method based on dc optimization is described. Numerical experience is reported, showing that the developed method allows one to solve in a few seconds problems with thousands of demand points.
\end{abstract}

Keywords: robustness, facility location, robust solutions, competitive location, Huff model, dc programming, branch and bound.

\section{Introduction}

The perception of the term robustness in the field of supply chain design, and, more specifically, in facility location, is wide. de Neufville (2004) defines robustness from the perspective of systems as "the ability of a system to maintain its operational capabilities under different circumstances", whereas Dong (2006) defines the robustness of a supply chain network as "the extent to which the network is able to carry out its functions despite some damage done to it, such as the removal of some of the nodes and/or links in a network."

The perception from the viewpoint of design from de Neufville (2004) and Dong (2006) comes close to the idea that robust means that a design

* This work has been funded by grants from the Spanish Ministry of Science and Innovation (TIN2008-01117,MTM2008-3032, MTM2009-14039), Junta de Andalucía (P08-TIC-3518,FQM-329), in part financed by the European Regional Development Fund (ERDF). Eligius Hendrix is a fellow of the Spanish "Ramón y Cajal" contract program, co-financed by the European Social Fund. 
performs under all circumstances. Robustness from this perspective is a measure of how robust a design is. What is generic here is that performance is seen from a YES/NO perspective. The design performs, works, fulfills specifications in a yes or no sense. If it always does, we perceive a design as robust. Formally, design $x$ is robust if $(x, w) \in Q \forall w \in W$, where the set $Q$ is a set of desired performance and $W$ is the set of outcomes of the uncertain parameter $w$. In other words, if $Q_{x}$ denotes the set of parameter values $w$ such that $(x, w) \in Q, x$ is seen as robust if $w \in Q_{x}$ for all $w \in W$.

In the literature on facility location, see e.g. Owen and Daskin (1998) and Snyder (2006), the design variable $x$ expresses usually locations of facilities, the uncertain parameters $w$ represent demand, buying power, population etc., and the set $Q$ is described with a threshold concept: a cost or a reward function $f(x, w)$ and a threshold value $\tau$ are given, and $Q$ is defined as the set

$$
Q=\{(x, w) \mid f(x, w) \# \tau\},
$$

where \# can be $<,>, \leq, \geq$.

Within this framework we can consider two main concepts of robustness, referred to in what follows as deviation robustness and probabilistic robustness.

In deviation robustness models, a nominal value $\mu$ of the uncertain parameter $w$ is given; for any feasible $x$, one can pose the question of how far deviations from the nominal value $\mu$ may go such that the design still performs as intended:

$$
R(x)=\min \left\{\|v-\mu\|: v \notin Q_{x}\right\},
$$

where $\|\cdot\|$ is a norm used to measure deviations in the parameter space $W$. Observe that, for any $x$ not performing properly for the nominal value $\mu$, i.e., $\mu \notin Q_{x}$, its robustness $R(x)$ is zero. The most robust solution, i.e., the solution $x$ with maximum value for $R(x)$, is sought. Such deviation robustness concept is called in Olieman (2008) the maximum inscribed sphere problem, as (2) means one wishes to find a maximum sized sphere of circumstances around the nominal value $\mu$ for which the design is still feasible. This concept has the advantage that no information is needed about the set of realisations, no probability distribution is required nor a range or worst-case outcome. It is thus well suited to problems with very high uncertainty, as happens, for instance, in long-term planning problems as those encountered in facility location.

Under particular forms of function $f$ in (1), a more tractable expression for $R$ can be derived. Indeed, as shown in Hendrix, Mecking and Hendriks (1996) and Carrizosa and Nickel (2003), if $f(x, w)$ is linear in $w$, i.e., if $f$ has the form

$$
f(x, w)=c(x)^{\top} w
$$

for a vector-valued function $c$, and \# is $>$, then $R(x)$ can be expressed as

$$
R(x)=\max \left\{\frac{c(x)^{\top} \mu-\tau}{\|c(x)\|^{\circ}}, 0\right\},
$$


where $\|\cdot\|^{\circ}$ denotes the norm dual to $\|\cdot\|$. For instance, if $\|\cdot\|$ is the $\ell_{p}$ norm, then $\|\cdot\|^{\circ}$ is the $\ell_{q}$ norm, with $1 / p+1 / q=1$.

Observe that, as soon as some $x^{*}$ exists with strictly positive robustness $R\left(x^{*}\right)$, maximizing $R$ turns out to be equivalent to maximizing $\bar{R}$, defined as

$$
\bar{R}(x)=\frac{c(x)^{\top} \mu-\tau}{\|c(x)\|^{\circ}} .
$$

Deviation robustness has been investigated in Carrizosa and Nickel (2003) within the field of continuous location for Weber problems. In such a problem, $\left\{p_{i}\right\}_{i \in I}$ is the set of demand points, which represent the geographical location of the customers, $f(x, w)$ is defined as the total transportation cost if a facility is located at $x$, and transportation costs to demand point $p_{i}$ are assumed to be proportional to the distance $d_{i}(x)$ between $x$ and demand point $p_{i}$. In other words, $f$ is assumed to have the form (3), with $c(x)=\left(c_{i}(x)\right)_{i \in I}, c_{i}(x)=d_{i}(x)$ and each $w_{i}$ is an uncertain parameter representing the demand of a demand point $p_{i}$, for which just a nominal value $\mu_{i}$ is given. Robustness, as defined in (4) is maximized via a finite-time convergent algorithm for particular models of distance functions $d_{i}$ and choices of $\|\cdot\|$. The reader is also referred to Hendrix et al. (1996) and Casado, Hendrix and García (2007) for applications of deviation robustness to other related problems.

Whereas deviation robustness can be seen as a worst-case measure, the concept of probabilistic robustness takes a probabilistic view, since it considers the circumstances $w$ as a random variable $\mathbf{w}$ and defines robustness as

$$
R(x)=P\{(x, \mathbf{w}) \in Q\} .
$$

In the literature on location, we see this robustness back under the terminology of "threshold" (without being called robustness) in Drezner, Drezner and Shioge (2002), who address a threshold model which maximizes the probability of reaching a minimum market share $\tau$

$$
R(x)=P\left\{c(x)^{\top} \mathbf{w} \geq \tau\right\},
$$

where the functions $c_{i}$ measure market share according to the Huff model, (Blanquero and Carrizosa 2009a, Drezner et al. 2002),

$$
c_{i}(x)=\frac{1}{1+h_{i} d_{i}^{\lambda}(x)},
$$

$d_{i}(x)$ is again the distance from demand point $p_{i}$ to a facility located at $x$, $\lambda \geq 1$ (typically $\lambda=2$ ) and $h_{i}$ is typically a positive constant that represents the relative attractiveness of competing facilities.

Even inspecting $R$ in (7) is, in general, very hard, since it involves multivariate calculus. In Drezner et al. (2002) it is assumed that w has a normal 
distribution with mean $\mu$ and covariance matrix $V$, and thus $R$ takes the simpler form

$$
R(x)=\Phi\left(\frac{c(x)^{\top} \mu-\tau}{\sqrt{c(x)^{\top} V c(x)}}\right),
$$

where $\Phi$ is the cumulative distribution function of the standard normal distribution. Hence, maximizing $R(x)$ is equivalent to maximizing the nonlinear fractional function $\frac{c(x)^{\top} \mu-\tau}{\sqrt{c(x)^{\top} V c(x)}}$.

The challenge in general with Huff-like models is that the market share functions $c_{i}$ in (8) are neither convex nor concave. Hence, optimizing $f(x)=$ $w^{\top} c(x)$ is a global optimization problem even for $w$ fixed. This is inherited by the threshold model (7), even under the assumption that $\mathbf{w}$ follows a normal distribution. In Drezner et al. (2002), a multistart strategy is used, thus no guarantee of having found the true global optimum is provided. As will be seen later, the probabilistic model (9) can be seen as a particular case of the deviation robustness model, for which a global optimization approach is proposed here.

The remainder of this paper is organized as follows. In Section 2 we introduce the problem of locating a competitive facility in the plane, where competition is described by a Huff-like model, and deviation robustness is to be maximized. It is shown in particular that the model of Drezner et al. (2002) appears as a particular case for a given choice of the norm $\|\cdot\|$.

The multimodal character of the optimization problem is investigated. Deterministic solution approaches that guarantee a global optimum solution are discussed in Section 3 . Numerical results are reported in Section 4. Finally we conclude in Section 5

\section{A competitive robustness location model}

We address the problem of locating a competitive facility with uncertain demand optimizing a deviation robustness criterion. Users are identified by an index $i \in I:=\{1,2, \ldots, N\}$, a demand location $p_{i}$, and a nominal value $\mu_{i}$ for the demand. Market is captured following a Huff model: The market captured by the facility at $x$ given demand $w$ is $f(x, w)=c(x)^{\top} w$, where $c$ is defined by (8).

The question which is answered is how far demand can fluctuate in a distance sense from its nominal value $\mu$ without capturing less than a given threshold value $\tau$. The robustness $R(x)$ of a facility located at $x$, to be maximized, is given by (4), or (5) if some $x^{*}$ exists with $R\left(x^{*}\right)>0$. No assumptions are made on the norm $\|\cdot\|$, and different choices of the norm lead to different models. We may take, for instance, the $\ell_{1}$ or $\ell_{\infty}$ norm to measure deviations with respect to the nominal value of the demand. Hence, for a given location $x, R(x)$ measures the maximum deviation (in the dual norm, $\ell_{\infty}$ or $\ell_{1}$ respectively) in the demand $w$ with respect to its nominal 
value $\mu$ such that the market captured remains above the threshold value $\tau$.

In particular, the stochastic programming formulation of Drezner et al. (2002) in (9) is a particular case of our model (5) by defining the norm $\|\cdot\|$ as

$$
\|w\|=\sqrt{w^{\top} V^{-1} w}
$$

which means that deviations with respect to the nominal vector of demands $\mu$ are measured by the dual $\|\cdot\|^{\circ}$ of $\|\cdot\|$,

$$
\|w\|^{\circ}=\sqrt{w^{\top} V w} .
$$

As the Huff-like continuous location problem is a global optimisation problem, one may expect that this structure is inherited by $R(x)$. The next research question is how multimodal is such model. We explore how parameters affect the number of local optima and we check the feasibility of nonlinear optimization local search to solve the optimization problem. The following repeatable experiment is carried out. A total of $m$ demand points is randomly generated on $[0,1]^{2}$ with generated demand $\mu$ from $[0,1]$ and $k$ competing facilities. The competing facilities give values to $h_{i}$ in (8) according to

$$
h_{i}=\sum_{j}^{k} \frac{1}{\delta_{i j}^{2}},
$$

where $\delta_{i j}$ is the Euclidean distance between demand point $p_{i}$ and existing facility $j$. The threshold was kept on $\tau=1$ and the norm $\|\cdot\|$ was the Euclidean norm for all experiments. The resulting objective function of a generated instance is depicted in Figure 1 having $k=3$ competitors.

To get a feeling for the multimodality of the problem we also generated the same instance, but then having $k=50$ competitors. As one can observe from Figure 2, the number of local maxima increases substantially. To investigate the trend for increasing number of demand points and competitors, we generated 50 intances for each setting varying the number of demand points as $m=40,200,1000$ and the number of competing facilities as $k=2,4,8,16,32$. Multistart using $10 \times k$ random starting points was applied for each generated instance to count the number of local optima found. FMINUNC was used to generate local optima. The average number of detected optima using this multistart strategy is given in Table 1 As can be observed, the number of optima depends mainly on the number of existing competing facilities.

A multistart strategy, as suggested by Drezner et al. (2002), can give us some confidence on the local optimum found. Indeed, if we knew we have about $m$ optima, and we can assume the region of attraction of the global optimum to occupy $\frac{100}{m} \%$ of the search space, then the probability to detect a global optimum after $r$ independent local searches is

$$
P=1-\left(\frac{m-1}{m}\right)^{r}
$$




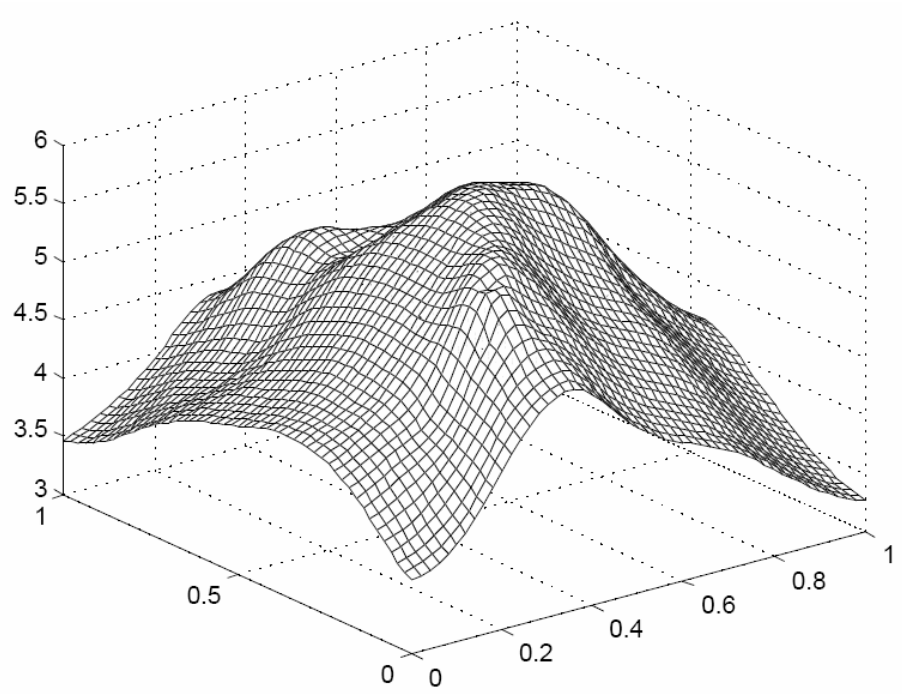

Fig. 1 Function $\bar{R}$ in $(5)$. Randomly generated instance, $m=200, k=3$

Table 1 Average number of optima over 50 instances, $k$ competitors $m$ demand points

\begin{tabular}{l|rrrrr}
$m \backslash k$ & 2 & 4 & 8 & 16 & 32 \\
\hline 40 & 2.76 & 3.74 & 6.76 & 13.88 & 26.12 \\
200 & 3.06 & 5.82 & 13.78 & 31.72 & 54.40 \\
1000 & 2.08 & 4.28 & 10.12 & 28.88 & 68.72
\end{tabular}

see for instance Hendrix and Toth (2010). Under these assumptions, if for example the number of local optima is $m=10$, we need $r=44$ trials to have a probability of $P=99 \%$ to reach the global optimum.

In other words, stochastic algorithms can reach, under some assumptions, a probabilistic target on effectiveness. Deterministic methods can be used to reach a guarantee on an accuracy of the reached optimum, Hendrix and Toth (2010). A specific method is elaborated in the next section.

\section{A deterministic solution method}

The basic idea in branch and bound methods consists of a recursive decomposition of the original problem into smaller disjoint subproblems until the solution is found. The method avoids visiting those subproblems which are known not to contain a solution. The initial set $T_{1}$ is subsequently partitioned in more and more refined subsets (branching) over which upper and lower bounds of an objective function value can be determined 


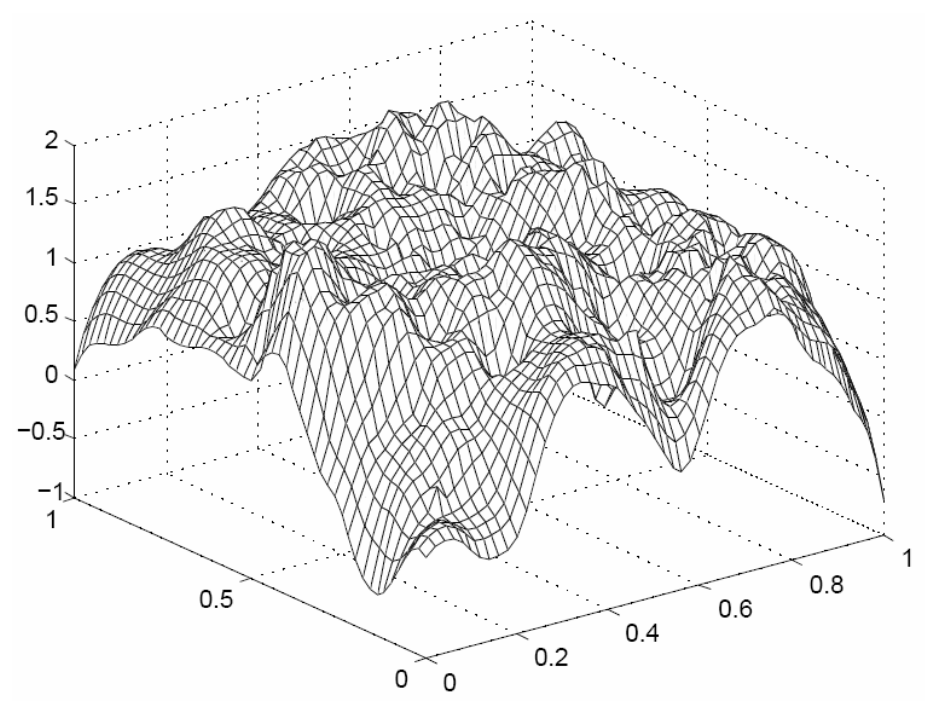

Fig. 2 Function $\bar{R}$ in 5 . Randomly generated instance, $m=200, k=50$

(bounding). In continuous location optimization, the most common branching procedures use rectangles and simplices (triangles). They are known in the literature as Big Square Small Square (BSSS), Hansen, Peeters, Richard and Thisse (1985), and Big Triangle Small Triangle (BTST), Drezner and Suzuki (2004).

To construct bounds, the classical approach in continuous location, already advocated in the seminal paper Hansen et al. (1985), exploits monotonicity and bounds derived with interval arithmetic. In recent years, alternative bounding schemes have been proposed in the literature of continuous location based on expressing the objective as a difference of two convex functions, see Drezner (2007), Blanquero and Carrizosa (2009a).

In this paper we observe that the objective is not only dc (it can be written as a difference of two convex functions), but it can be written in terms of compositions of convex and convex monotonic functions. In the terminology of Blanquero and Carrizosa (2009a), the functions involved are dcm functions (difference of convex monotonic), and thus the bounding strategies developed in Blanquero and Carrizosa (2009a) can be used here.

The following key result, stated in Bello, Blanquero and Carrizosa (2009) and with straightforward proof, enables one to express the function in (8) as difference of convex monotonic functions.

Proposition 1 Given $h>0, \lambda \geq 1$, define $d_{0}$ as

$$
d_{0}=\left(\frac{\lambda-1}{h(\lambda+1)}\right)^{\frac{1}{\lambda}}
$$


and the functions $\Phi, \Phi^{1}, \Phi^{2}: \mathbb{R}_{+} \longrightarrow \mathbb{R}$ as

$$
\begin{aligned}
\Phi(d) & =\frac{1}{1+h d^{\lambda}} \\
\Phi^{1}(d) & = \begin{cases}\Phi\left(d_{0}\right)+\Phi^{\prime}\left(d_{0}\right)\left(d-d_{0}\right) & \text { if } d \leq d_{0} \\
\Phi(d) & \text { if } d>d_{0}\end{cases} \\
\Phi^{2}(d) & = \begin{cases}\Phi\left(d_{0}\right)+\Phi^{\prime}\left(d_{0}\right)\left(d-d_{0}\right)-\Phi(d) & \text { if } d \leq d_{0} \\
0 & \text { if } d>d_{0}\end{cases}
\end{aligned}
$$

One has:

1. $\Phi=\Phi^{1}-\Phi^{2}$

2. $\Phi^{1}, \Phi^{2}$ are smooth convex nonincreasing functions in $\mathbb{R}_{+}$

Define

$$
\begin{aligned}
\Phi_{i}(d) & =\frac{1}{1+h_{i} d^{\lambda}}, \quad i=1,2, \ldots, N \\
\Psi\left(d_{1}, \ldots, d_{N}\right) & =\left(\Phi_{1}\left(d_{1}\right), \ldots, \Phi_{N}\left(d_{N}\right)\right) .
\end{aligned}
$$

Observe that

$$
c(x)=\Psi\left(d_{1}(x), \ldots, d_{N}(x)\right) .
$$

By Proposition 1, we can express the function $\Psi\left(d_{1}, \ldots, d_{N}\right)^{\top} \mu-\tau$ as a difference of convex nonincreasing functions in $\mathbb{R}_{+}$. We follow Blanquero and Carrizosa (2009a) to obtain bounds by respectively majorizing by a convex function the numerator and minorizing by a concave function the denominator in (4) on a given polytope $T$ in $\mathbb{R}^{2}$.

First, one easily obtains a convex function $U$ such that

$$
c(x)^{\top} \mu-\tau \leq U(x) \quad \forall x \in T .
$$

Indeed, since, by assumption, each $d_{i}$ is convex, if $x_{i}^{*}$ is an arbitrary point of $T$ and $\xi_{i}$ is a subgradient of $d_{i}$ at $x_{i}^{*}$, one has

$$
d_{i}(x) \geq d_{i}\left(x_{i}^{*}\right)+\xi_{i}^{\top}\left(x-x_{i}^{*}\right) \quad \forall x \in T .
$$

Since $\Phi_{i}^{1}$ is nonincreasing, one has by 17 that

$$
\Phi_{i}^{1}\left(d_{i}(x)\right) \leq \Phi_{i}^{1}\left(d_{i}\left(x_{i}^{*}\right)+\xi_{i}^{\top}\left(x-x_{i}^{*}\right)\right) \quad \forall x \in T .
$$

Observe also that the right-hand side function in $(18)$ is the composition of a convex and an affine function, and it is thus convex.

On the other hand, $\Phi_{i}^{2}$ is a convex smooth function. Hence thus

$$
\Phi_{i}^{2}(d) \geq \Phi_{i}^{2}\left(d_{i}\left(x_{i}^{*}\right)\right)+\left(\Phi_{i}^{2}\left(d_{i}\left(x_{i}^{*}\right)\right)\right)^{\prime}\left(d-d_{i}\left(x_{i}^{*}\right)\right) \quad \forall d \geq 0,
$$

thus

$$
\Phi_{i}^{2}\left(d_{i}(x)\right) \geq \Phi_{i}^{2}\left(d_{i}\left(x_{i}^{*}\right)\right)+\left(\Phi_{i}^{2}\left(d_{i}\left(x_{i}^{*}\right)\right)\right)^{\prime}\left(d_{i}(x)-d_{i}\left(x_{i}^{*}\right)\right) \quad \forall x \in T .
$$


Moreover, $\Phi_{i}^{2}$ is nonincreasing, $\left(\Phi_{i}^{2}\left(d_{i}\left(x_{i}^{*}\right)\right)\right)^{\prime} \leq 0$, thus the right-hand side function in $(19)$ is concave.

Joining (18) and 20) we have that

$\Phi_{i}^{1}\left(d_{i}(x)-\Phi_{i}^{2}\left(d_{i}(x)\right) \leq U(x):=\Phi_{i}^{1}\left(d_{i}\left(x_{i}^{*}\right)+\xi_{i}^{\top}\left(x-x_{i}^{*}\right)\right)-\Phi_{i}^{2}\left(d_{i}\left(x_{i}^{*}\right)\right)-\left(\Phi_{i}^{2}\left(d_{i}\left(x_{i}^{*}\right)\right)^{\prime}\left(d_{i}(x)-d_{i}\left(x_{i}^{*}\right)\right)\right.\right.$,

and $U$ is convex in $T$.

Let us consider now the denominator. The following proposition is a consequence of the results in Blanquero and Carrizosa (2000) and Blanquero and Carrizosa (2009b)

Proposition 2 Given a norm $\|\cdot\|$, the functions $\left.x \in \mathbb{R}^{2} \longmapsto \| c_{(} x\right) \|$ and $d \longmapsto\|\Psi(d)\|$ can be expressed as the difference of convex functions. Moreover, if the norm $\|\cdot\|$ is monotonic in the positive orthant, then the function $d \longmapsto\|\Psi(d)\|$ can be expressed as the difference of two convex nonincreasing functions.

Hence, given a polytope $T$ in the plane, we can find a concave function $L$ such that

$$
\|c(x)\| \geq L(x) \quad \forall x \in T .
$$

Note that $\ell_{p}$ norms satisfy the monotonicity assumptions on $\|\cdot\|$. This assumption also holds for the norm (10) as soon as V is a matrix of nonnegative elements, i.e., that the demand at different users are positively correlated. However, the monotonicity assumption does not hold for arbitrary norms, and thus for arbitrary norms, or, for instance, for norm (10) with negative correlations, the weaker result should be used. Nevertheless Drezner et al. (2002) claim that "it is likely that the distributions of buying power at two demand points are positively correlated. This might be due to good economic conditions or other factors resulting in either higher or lower than expected buying power in any community." Hence, the strongest assumptions seem to be applicable also in real world problems.

If we can guarantee that $L(x)>0$ for all $x \in T$, then $U / L$ is the ratio of a convex over a positive concave function, thus it is quasiconvex. This implies it attains its maximum at extreme points on $T$, i.e.,

$$
\frac{c(x)^{\top} \mu-\tau}{\|c(x)\|} \leq \frac{U(x)}{L(x)} \leq \max _{v \in \operatorname{ext}(T)} \frac{U(v)}{L(v)},
$$

and thus

$$
R(x) \leq \max \left\{0, \max _{v \in \operatorname{ext}(T)} \frac{U(v)}{L(v)}\right\} .
$$

Hence, we have an upper bound for the objective as soon as we can asssert that $L(x)>0$ for all $x \in T$. Since $L$ is concave on $T$, such a condition is equivalent to

$$
\min _{v \in \operatorname{ext}(T)} L(v)>0
$$




\section{Computational experiments}

In this section we show how the bounding scheme outlined in Section 3 can be used to solve the problem

$$
\max _{x \in S} R(x)
$$

where $R(x)$ is given by (4) under the assumptions described in Section 2. The bounding strategy based on dcm functions here applied has been successfully used to solve other nonconvex location problems, see Blanquero and Carrizosa (2009a) and Bello et al. (2009).

Instances of $(26)$ are generated in the following way:

- The feasible region is assumed to be the unit square $S=[0,1] \times[0,1]$.

- There are 10 existing facilities, with locations randomly and uniformly distributed in $S$.

- Demand points are also randomly and uniformly generated in $S$. The number $N$ of demand points ranges from very small $(N=10)$ to large $(N=10000)$.

For each number $N$ of demand points, a nominal vector $\mu$ was randomly generated in $[0,1]^{N}$, and the optimal objective value $z_{\text {nom }}$ of the problem $\max _{x \in S} c(x)^{\top} \mu$ was computed. Observe that in order to compute $z_{\text {nom }}$, a global optimization problem is solved. The optimization problem (26) was solved using different values of the threshold, ranging from $0.3 z_{n o m}$ to $1.5 z_{n o m}$ with a step of $0.05 z_{n o m}$. The norm $\|\cdot\|$ considered in 26$)$ was the Euclidean.

For each choice of $N$ and $\tau$, ten instances were generated and solved using the BSSS method. The program code was written in Fortran, compiled by Intel Fortran 10.1 and ran on a $2.4 \mathrm{GHz}$ computer under Windows XP. The solutions were found to an accuracy of $10^{-8}$.

The bounds for the numerator of $R(x)$ were computed according to the procedure proposed in Blanquero and Carrizosa (2009a), as has been detailed in Section 3. Regarding the denominator, a dcm decomposition for it was obtained combining the same procedure with Theorem 1 in Blanquero and Carrizosa (2009b), since the norm considered is monotonic in $\mathbb{R}_{+}^{N}$. The bounds obtained using this result are better than those provided by Proposition 1.1 in Blanquero and Carrizosa (2000), which could have also been used.

Tables 2 to 11 report, for the different threshold values $\tau$ and number $N$ of demand points, statistics on the number of iterations, the memory usage, measured via the maximum size of the list of squares to be inspected in the branch and bound, and the CPU time.

The numerical results for different threshold values $\tau$ are shown in Tables where one can observe that the computational effort needed to solve the problem decreases as soon as the threshold value grows, especially when 
the threshold exceeds $z_{n o m}$, since in that case $R(x)=0 \quad \forall x \in S$, and the algorithm quickly closes the gap.

Average CPU time and number of iterations for different number of demand points is shown in Figures 3 and 4 for different threshold values $\tau$. For the instances with positive robustness, i.e., with $\tau<z_{\text {nom }}$, the average running times and number of iterations increase at most linearly in the number $N$ of demand points. Finally, Figure 5 illustrates the evolution of the average $\mathrm{CPU}$ time as a function of the threshold value, for $N$ equal to $100,1000,5000$ and 10000 .

\begin{tabular}{|c|c|c|c|c|c|c|c|c|c|}
\hline \multirow[t]{2}{*}{$N$} & \multicolumn{3}{|c|}{ Iterations } & \multicolumn{3}{|c|}{ Max squares } & \multicolumn{2}{|c|}{ Time (s) } & \\
\hline & Min & Max & Ave & $\overline{\text { Min }}$ & Max & Ave & Min & Max & Ave \\
\hline 10 & 157 & 393 & 268,50 & 22 & 64 & 45,10 & 0,000 & 0,016 & 0,008 \\
\hline 20 & 198 & 291 & 240,90 & 31 & 53 & 40,80 & 0,000 & 0,016 & 0,013 \\
\hline 50 & 242 & 472 & 328,00 & 44 & 78 & 58,80 & 0,016 & 0,047 & 0,030 \\
\hline 100 & 316 & 1100 & 435,70 & 71 & 176 & 92,90 & 0,047 & 0,203 & 0,081 \\
\hline 200 & 339 & 550 & 412,30 & 78 & 133 & 103,70 & 0,125 & 0,219 & 0,153 \\
\hline 500 & 367 & 1347 & 635,20 & 108 & 195 & 150,00 & 0,344 & 1,250 & 0,588 \\
\hline 1000 & 397 & 1116 & 562,60 & 93 & 203 & 160,90 & 0,719 & 2,063 & 1,036 \\
\hline 2000 & 434 & 1273 & 662,40 & 145 & 258 & 184,20 & 1,594 & 4,719 & 2,445 \\
\hline 5000 & 526 & 2136 & 847,10 & 192 & 357 & 283,60 & 4,906 & 19,891 & 7,834 \\
\hline 10000 & 713 & 3854 & 1724,90 & 305 & 570 & 396,70 & 13,188 & 71,328 & 31,888 \\
\hline
\end{tabular}

Table 2 Computational results for $\tau=0.3 z_{\text {nom }}$

\begin{tabular}{|c|c|c|c|c|c|c|c|c|c|}
\hline \multirow[t]{2}{*}{$N$} & \multicolumn{3}{|c|}{ Iterations } & \multicolumn{3}{|c|}{ Max squares } & \multicolumn{3}{|c|}{ Time (s) } \\
\hline & Min & Max & Ave & Min & $\operatorname{Max}$ & Ave & Min & Max & Ave \\
\hline 10 & 160 & 542 & 276,20 & 21 & 101 & 45,60 & 0,000 & 0,016 & 0,003 \\
\hline 20 & 182 & 338 & 228,40 & 25 & 56 & 39,70 & 0,000 & 0,016 & 0,009 \\
\hline 50 & 240 & 626 & 341,80 & 38 & 101 & 55,70 & 0,016 & 0,063 & 0,030 \\
\hline 100 & 294 & 551 & 387,10 & 57 & 103 & 77,30 & 0,047 & 0,094 & 0,069 \\
\hline 200 & 338 & 724 & 459,30 & 63 & 142 & 95,50 & 0,125 & 0,266 & 0,170 \\
\hline 500 & 353 & 1237 & 670,60 & 96 & 187 & 138,20 & 0,328 & 1,156 & 0,619 \\
\hline 1000 & 339 & 1168 & 661,40 & 79 & 216 & 159,40 & 0,625 & 2,172 & 1,223 \\
\hline 2000 & 455 & 1293 & 728,30 & 136 & 283 & 184,20 & 1,688 & 4,781 & 2,686 \\
\hline 5000 & 544 & 3198 & 1303,50 & 182 & 483 & 293,30 & 5,016 & 29,672 & 12,075 \\
\hline 10000 & 800 & 3204 & 1556,90 & 252 & 438 & 352,10 & 14,766 & 59,344 & 28,794 \\
\hline
\end{tabular}

Table 3 Computational results for $\tau=0.4 z_{\text {nom }}$

\section{Conclusion}

Two different robustness concepts, deviation robustness and probabilistic, are described. The deviation concept has been elaborated in a new generic 


\begin{tabular}{|c|c|c|c|c|c|c|c|c|c|}
\hline \multirow[t]{2}{*}{$N$} & \multicolumn{3}{|c|}{ Iterations } & \multicolumn{3}{|c|}{ Max squares } & \multicolumn{2}{|c|}{ Time $(\mathrm{s})$} & \multirow[b]{2}{*}{ Ave } \\
\hline & Min & Max & Ave & Min & $\operatorname{Max}$ & Ave & Min & Max & \\
\hline 10 & 155 & 484 & 239,20 & 22 & 88 & 38,80 & 0,000 & 0,016 & 0,008 \\
\hline 20 & 160 & 1147 & 306,70 & 23 & 255 & 56,80 & 0,000 & 0,031 & 0,013 \\
\hline 50 & 223 & 722 & 373,30 & 31 & 137 & 64,90 & 0,016 & 0,063 & 0,036 \\
\hline 100 & 271 & 448 & 321,10 & 43 & 88 & 65,10 & 0,047 & 0,078 & 0,059 \\
\hline 200 & 305 & 810 & 474,90 & 47 & 146 & 89,60 & 0,125 & 0,297 & 0,178 \\
\hline 500 & 362 & 1339 & 662,10 & 70 & 258 & 134,60 & 0,328 & 1,250 & 0,616 \\
\hline 1000 & 355 & 1355 & 838,30 & 75 & 206 & 150,40 & 0,656 & 2,500 & 1,550 \\
\hline 2000 & 486 & 1111 & 757,90 & 96 & 238 & 164,20 & 1,797 & 4,125 & 2,795 \\
\hline 5000 & 627 & 2022 & 1254,80 & 197 & 333 & 258,20 & 5,781 & 18,750 & 11,605 \\
\hline 10000 & 751 & 3185 & 1670,50 & 193 & 509 & 331,30 & 13,859 & 58,844 & 30,883 \\
\hline
\end{tabular}

Table 4 Computational results for $\tau=0.5 z_{n o m}$

\begin{tabular}{|c|c|c|c|c|c|c|c|c|c|}
\hline \multirow[t]{2}{*}{$N$} & \multicolumn{3}{|c|}{ Iterations } & \multicolumn{3}{|c|}{ Max squares } & \multicolumn{2}{|c|}{ Time (s) } & \\
\hline & Min & Max & Ave & $\overline{\operatorname{Min}}$ & Max & Ave & Min & $\operatorname{Max}$ & Ave \\
\hline 10 & 136 & 430 & 210,40 & 20 & 84 & 34,40 & 0,000 & 0,016 & 0,005 \\
\hline 20 & 130 & 723 & 247,30 & 19 & 158 & 42,90 & 0,000 & 0,031 & 0,013 \\
\hline 50 & 177 & 528 & 294,50 & 25 & 93 & 48,50 & 0,016 & 0,047 & 0,028 \\
\hline 100 & 215 & 450 & 303,20 & 30 & 87 & 54,90 & 0,047 & 0,094 & 0,058 \\
\hline 200 & 260 & 492 & 386,90 & 34 & 93 & 62,60 & 0,094 & 0,172 & 0,142 \\
\hline 500 & 391 & 4166 & 923,70 & 51 & 987 & 180,00 & 0,359 & 3,844 & 0,856 \\
\hline 1000 & 544 & 2427 & 907,10 & 81 & 570 & 168,90 & 1,000 & 4,500 & 1,681 \\
\hline 2000 & 596 & 1458 & 1011,70 & 75 & 215 & 160,40 & 2,203 & 5,375 & 3,744 \\
\hline 5000 & 991 & 2446 & 1324,40 & 127 & 434 & 239,40 & 9,188 & 22,594 & 12,241 \\
\hline 10000 & 916 & 3034 & 1916,40 & 143 & 447 & 280,90 & 16,891 & 56,047 & 35,456 \\
\hline
\end{tabular}

Table 5 Computational results for $\tau=0.6 z_{n o m}$

\begin{tabular}{|c|c|c|c|c|c|c|c|c|c|}
\hline \multirow[t]{2}{*}{$N$} & \multicolumn{3}{|c|}{ Iterations } & \multicolumn{3}{|c|}{ Max squares } & \multicolumn{3}{|c|}{ Time (s) } \\
\hline & Min & Max & Ave & $\overline{\text { Min }}$ & Max & Ave & Min & Max & Ave \\
\hline 10 & 123 & 371 & 193,70 & 19 & 71 & 32,40 & 0,000 & 0,016 & 0,005 \\
\hline 20 & 117 & 369 & 201,10 & 16 & 70 & 32,30 & 0,000 & 0,016 & 0,009 \\
\hline 50 & 156 & 440 & 232,80 & 23 & 74 & 37,60 & 0,016 & 0,047 & 0,022 \\
\hline 100 & 192 & 380 & 244,60 & 29 & 57 & 41,60 & 0,031 & 0,078 & 0,045 \\
\hline 200 & 229 & 488 & 314,80 & 32 & 71 & 48,20 & 0,078 & 0,172 & 0,117 \\
\hline 500 & 306 & 779 & 448,30 & 41 & 130 & 69,60 & 0,281 & 0,719 & 0,416 \\
\hline 1000 & 465 & 655 & 571,80 & 68 & 157 & 93,80 & 0,859 & 1,203 & 1,058 \\
\hline 2000 & 482 & 1055 & 795,40 & 63 & 219 & 125,20 & 1,797 & 3,906 & 2,947 \\
\hline 5000 & 647 & 1400 & 1031,40 & 89 & 248 & 167,60 & 6,000 & 12,891 & 9,542 \\
\hline 10000 & 834 & 2169 & 1361,30 & 112 & 337 & 183,60 & 15,563 & 40,016 & 25,181 \\
\hline
\end{tabular}

Table 6 Computational results for $\tau=0.7 z_{\text {nom }}$ 


\begin{tabular}{|c|c|c|c|c|c|c|c|c|c|}
\hline \multirow[t]{2}{*}{$N$} & \multicolumn{3}{|c|}{ Iterations } & \multicolumn{3}{|c|}{ Max squares } & \multicolumn{2}{|c|}{ Time $(\mathrm{s})$} & \multirow[b]{2}{*}{ Ave } \\
\hline & Min & Max & Ave & $\overline{\operatorname{Min}}$ & $\operatorname{Max}$ & Ave & Min & Max & \\
\hline 10 & 117 & 403 & 191,00 & 18 & 84 & 33,00 & 0,000 & 0,016 & 0,005 \\
\hline 20 & 103 & 243 & 165,60 & 15 & 45 & 27,50 & 0,000 & 0,016 & 0,009 \\
\hline 50 & 126 & 531 & 221,10 & 19 & 103 & 37,70 & 0,000 & 0,047 & 0,023 \\
\hline 100 & 150 & 514 & 231,30 & 21 & 100 & 38,00 & 0,031 & 0,094 & 0,044 \\
\hline 200 & 179 & 301 & 231,50 & 24 & 46 & 35,60 & 0,063 & 0,109 & 0,086 \\
\hline 500 & 243 & 706 & 359,20 & 33 & 101 & 54,60 & 0,234 & 0,656 & 0,339 \\
\hline 1000 & 335 & 721 & 472,60 & 45 & 130 & 73,10 & 0,609 & 1,391 & 0,881 \\
\hline 2000 & 376 & 795 & 525,90 & 49 & 149 & 81,20 & 1,406 & 2,938 & 1,948 \\
\hline 5000 & 445 & 1766 & 812,40 & 58 & 265 & 118,10 & 4,141 & 16,359 & 7,509 \\
\hline 10000 & 555 & 1352 & 856,60 & 72 & 179 & 113,00 & 10,344 & 25,016 & 15,869 \\
\hline
\end{tabular}

Table 7 Computational results for $\tau=0.8 z_{\text {nom }}$

\begin{tabular}{|c|c|c|c|c|c|c|c|c|c|}
\hline \multirow[t]{2}{*}{$N$} & \multicolumn{3}{|c|}{ Iterations } & \multicolumn{3}{|c|}{ Max squares } & \multicolumn{3}{|c|}{ Time $(\mathrm{s})$} \\
\hline & Min & Max & Ave & $\overline{\text { Min }}$ & Max & Ave & Min & Max & Ave \\
\hline 10 & 106 & 612 & 196,10 & 16 & 156 & 38,60 & 0,000 & 0,000 & 0,000 \\
\hline 20 & 93 & 206 & 139,60 & 15 & 32 & 21,50 & 0,000 & 0,016 & 0,008 \\
\hline 50 & 108 & 288 & 156,10 & 15 & 47 & 24,40 & 0,016 & 0,016 & 0,016 \\
\hline 100 & 119 & 580 & 201,80 & 17 & 137 & 36,00 & 0,016 & 0,109 & 0,039 \\
\hline 200 & 144 & 239 & 183,10 & 21 & 39 & 27,60 & 0,047 & 0,094 & 0,069 \\
\hline 500 & 190 & 443 & 260,60 & 28 & 65 & 39,00 & 0,172 & 0,406 & 0,242 \\
\hline 1000 & 245 & 500 & 332,80 & 32 & 87 & 51,80 & 0,453 & 0,922 & 0,616 \\
\hline 2000 & 253 & 523 & 359,60 & 31 & 101 & 52,80 & 0,938 & 1,938 & 1,331 \\
\hline 5000 & 318 & 1081 & 529,00 & 45 & 165 & 74,10 & 3,000 & 9,984 & 4,895 \\
\hline 10000 & 372 & 852 & 531,80 & 50 & 109 & 69,10 & 6,922 & 15,734 & 9,853 \\
\hline
\end{tabular}

Table 8 Computational results for $\tau=0.9 z_{\text {nom }}$

\begin{tabular}{|c|c|c|c|c|c|c|c|c|c|}
\hline \multirow[t]{2}{*}{$N$} & \multicolumn{3}{|c|}{ Iterations } & \multicolumn{3}{|c|}{ Max squares } & \multicolumn{3}{|c|}{ Time (s) } \\
\hline & Min & $\operatorname{Max}$ & Ave & $\overline{M i n}$ & Max & Ave & Min & $\operatorname{Max}$ & Ave \\
\hline 10 & 90 & 234 & 142,20 & 12 & 46 & 23,60 & 0,000 & 0,016 & 0,002 \\
\hline 20 & 83 & 145 & 115,40 & 12 & 26 & 17,00 & 0,000 & 0,016 & 0,003 \\
\hline 50 & 94 & 226 & 130,80 & 14 & 37 & 20,20 & 0,000 & 0,016 & 0,006 \\
\hline 100 & 99 & 210 & 138,40 & 14 & 38 & 20,00 & 0,016 & 0,031 & 0,023 \\
\hline 200 & 115 & 190 & 140,10 & 15 & 29 & 19,50 & 0,031 & 0,078 & 0,052 \\
\hline 500 & 149 & 239 & 179,70 & 16 & 37 & 25,00 & 0,141 & 0,219 & 0,167 \\
\hline 1000 & 174 & 295 & 208,20 & 22 & 41 & 29,10 & 0,328 & 0,547 & 0,386 \\
\hline 2000 & 163 & 393 & 230,10 & 19 & 59 & 32,00 & 0,594 & 1,453 & 0,853 \\
\hline 5000 & 181 & 440 & 273,50 & 25 & 73 & 37,70 & 1,672 & 4,078 & 2,533 \\
\hline 10000 & 164 & 388 & 244,40 & 24 & 50 & 32,10 & 3,063 & 7,156 & 4,533 \\
\hline
\end{tabular}

Table 9 Computational results for $\tau=1.0 z_{\text {nom }}$ 

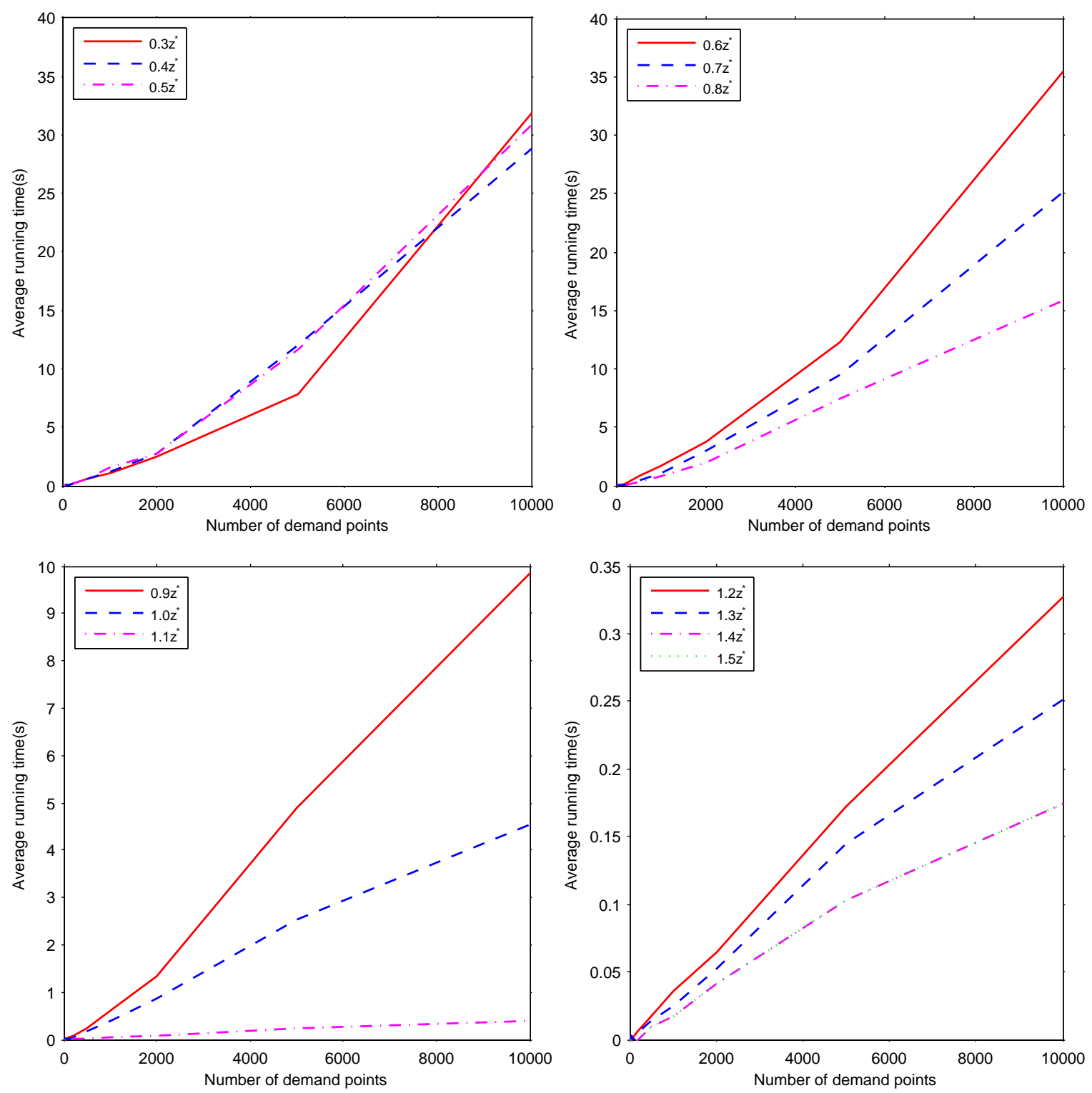

Fig. 3 Average running times versus number of demand points for different thresholds 

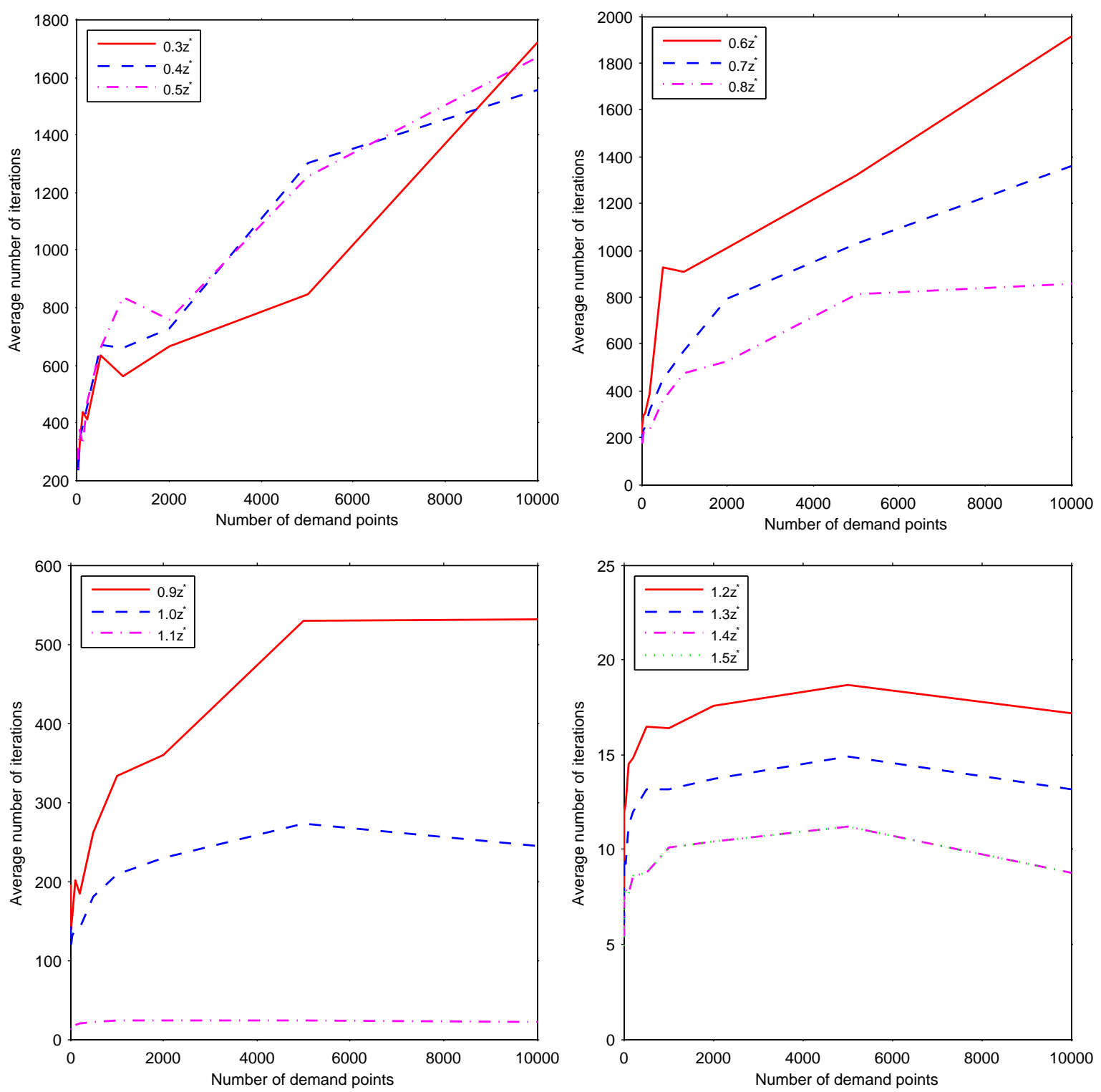

Fig. 4 Average number of iterations versus number of demand points for different thresholds 


\begin{tabular}{|c|c|c|c|c|c|c|c|c|c|}
\hline \multirow[t]{2}{*}{$N$} & \multicolumn{3}{|c|}{ Iterations } & \multicolumn{3}{|c|}{ Max squares } & \multicolumn{3}{|c|}{ Time (s) } \\
\hline & Min & $\operatorname{Max}$ & Ave & $\overline{\text { Min }}$ & Max & Ave & Min & Max & Ave \\
\hline 10 & 8 & 19 & 11,50 & 3 & 7 & 4,50 & 0,000 & 0,000 & 0,000 \\
\hline 20 & 10 & 25 & 18,00 & 4 & 10 & 6,70 & 0,000 & 0,000 & 0,000 \\
\hline 50 & 11 & 24 & 17,90 & 4 & 10 & 7,30 & 0,000 & 0,016 & 0,002 \\
\hline 100 & 13 & 33 & 18,80 & 5 & 12 & 7,50 & 0,000 & 0,016 & 0,005 \\
\hline 200 & 12 & 26 & 20,00 & 5 & 10 & 8,50 & 0,000 & 0,016 & 0,008 \\
\hline 500 & 15 & 28 & 22,30 & 5 & 12 & 9,00 & 0,016 & 0,031 & 0,023 \\
\hline 1000 & 16 & 35 & 23,70 & 8 & 11 & 9,10 & 0,031 & 0,063 & 0,045 \\
\hline 2000 & 17 & 34 & 24,40 & 9 & 14 & 10,20 & 0,063 & 0,141 & 0,092 \\
\hline 5000 & 15 & 36 & 24,50 & 6 & 11 & 9,30 & 0,141 & 0,328 & 0,228 \\
\hline 10000 & 14 & 28 & 21,40 & 5 & 10 & 8,80 & 0,266 & 0,516 & 0,400 \\
\hline
\end{tabular}

Table 10 Computational results for $\tau=1.1 z_{n o m}$

\begin{tabular}{|c|c|c|c|c|c|c|c|c|c|}
\hline \multirow[t]{2}{*}{$N$} & \multicolumn{3}{|c|}{ Iterations } & \multicolumn{3}{|c|}{ Max squares } & \multicolumn{3}{|c|}{ Time (s) } \\
\hline & Min & $\operatorname{Max}$ & Ave & $\overline{\text { Min }}$ & Max & Ave & Min & Max & Ave \\
\hline 10 & 5 & 14 & 8,00 & 2 & 5 & 3,80 & 0,000 & 0,000 & 0,000 \\
\hline 20 & 7 & 16 & 12,00 & 3 & 7 & 5,10 & 0,000 & 0,016 & 0,002 \\
\hline 50 & 7 & 17 & 12,40 & 4 & 8 & 5,60 & 0,000 & 0,000 & 0,000 \\
\hline 100 & 7 & 20 & 14,50 & 4 & 9 & 6,40 & 0,000 & 0,016 & 0,002 \\
\hline 200 & 10 & 18 & 14,80 & 5 & 9 & 7,40 & 0,000 & 0,016 & 0,006 \\
\hline 500 & 10 & 20 & 16,50 & 5 & 10 & 7,60 & 0,016 & 0,031 & 0,017 \\
\hline 1000 & 10 & 21 & 16,40 & 5 & 10 & 7,70 & 0,016 & 0,047 & 0,036 \\
\hline 2000 & 12 & 21 & 17,60 & 7 & 10 & 8,50 & 0,047 & 0,078 & 0,064 \\
\hline 5000 & 14 & 21 & 18,70 & 5 & 10 & 8,50 & 0,125 & 0,203 & 0,172 \\
\hline 10000 & 10 & 21 & 17,20 & 4 & 10 & 8,10 & 0,188 & 0,406 & 0,327 \\
\hline
\end{tabular}

Table 11 Computational results for $\tau=1.2 z_{n o m}$

robust competitive continuous location model. We show it also captures stochastic programming models that follow the probabilistic approach.

The model inherits the multimodal character of the underlying Huffmodel. We found that the number of optima for such a model mainly depends on the number of existing competitive facilities and it does not increase substantially with the number of demand points.

A branch and bound approach gives a guaranteed global optimum of a competitive location model. The computational experiments reported support the idea that using dc-programming techniques enables one to solve problems with thousands of demand points in a few seconds.

\section{References}

Bello, L., Blanquero, R. and Carrizosa, E.: 2009, Minimum-regret Huff location models, Technical report, Universidad de Sevilla.

Blanquero, R. and Carrizosa, E.: 2000, Optimization of the norm of a vectorvalued dc function and applications, Journal of Optimization Theory and Applications 107, 245-260. 

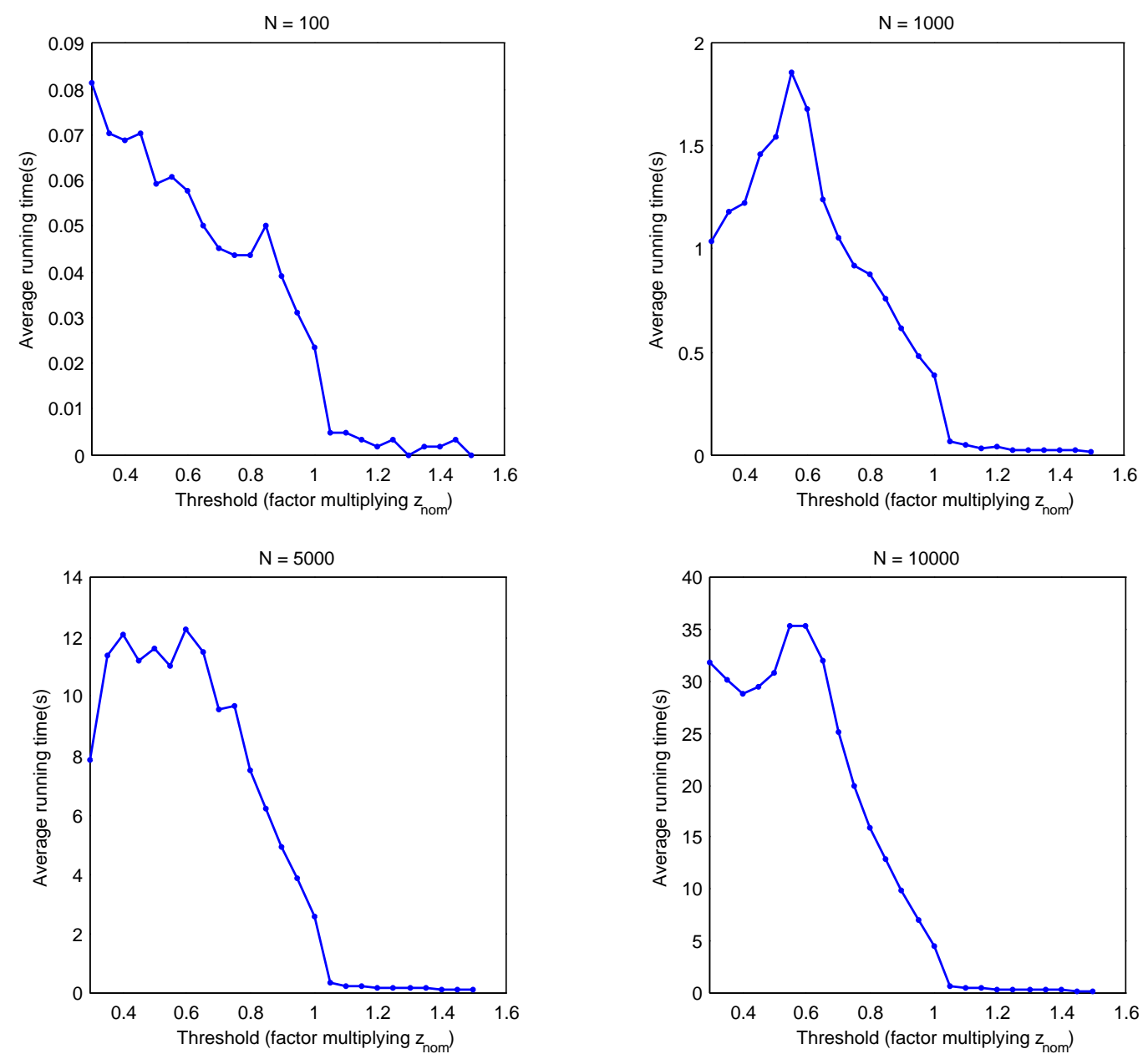

Fig. 5 Average running times versus thresholds for different number of demand points

Blanquero, R. and Carrizosa, E.: 2009a, Continuous location problems and big triangle small triangle: Constructing better bounds, Journal of Global Optimization 45, 389-402.

Blanquero, R. and Carrizosa, E.: 2009b, On the norm of a dc function, Journal of Global Optimization. DOI: 10.1007/s10898-009-9487-y.

Carrizosa, E. and Nickel, S.: 2003, Robust facility location, Mathematical Methods of Operations Research 58, 331-349.

Casado, L., Hendrix, E. and García, I.: 2007, Infeasibility spheres for finding robust solutions of blending problems with quadratic constraints, Journal of Global Optimization 39(4), 577-593.

de Neufville, R.: 2004, Uncertainty management for engineering systems planning and design, Engineering Systems Monograph . 
Dong, M.: 2006, Development of supply chain network robustness index, Int. J. Services Operations and Informatics 58, 54-66.

Drezner, T., Drezner, Z. and Shioge, S.: 2002, A threshold-satisfying competitive location model, Journal of Regional Science 42 (2), 287-299.

Drezner, Z.: 2007, A general global optimization approach for solving location problems in the plane, Journal of Global Optimization 37, 305-319.

Drezner, Z. and Suzuki, A.: 2004, The Big Triangle Small Triangle method for the solution of nonconvex facility location problems, Operations Research 52, 128135.

Hansen, P., Peeters, D., Richard, D. and Thisse, J.-F.: 1985, The minisum and minimax location problems revisited, Operations Research 33, 1251-1265.

Hendrix, E. M. T., Mecking, C. J. and Hendriks, T. H. B.: 1996, Finding robust solutions for product design problems, European Journal of Operational Research 92, 28-36.

Hendrix, E. M. and Toth, B. G.: 2010, Introduction to Nonlinear and Global Optimization, Springer, Cambridge.

Olieman, N. J.: 2008, Methods for Robustness Programming, Wageningen University, Wageningen, http://www.library.wur.nl/wda/dissertations/dis4405.pdf.

Owen, S. H. and Daskin, M. S.: 1998, Strategic facility location: A review, European Journal of Operational Research 111, 423-447.

Snyder, L. V.: 2006, Facility location under uncertainty: a review, IIE Transactions 38, 537-554. 